CERN-TH.6676/92

SOME RESULTS FOR STRINGS AT $D>1^{1}$

\author{
Luis Alvarez-Gaumé \\ Theory Division \\ CERN \\ CH-1211 Geneva 23 \\ Switzerland
}

CERN-TH.6676/92

October 1992

\footnotetext{
${ }^{1}$ Talk presented at the XXVI Rochester Conference in High Energy Physics, Dallas, August 4-13, 1992.
} 


\title{
SOME RESULTS FOR STRINGS AT $D>1$
}

\author{
Luis Alvarez-Gaumé \\ Theory Division, CERN \\ CH - 1211 Geneva 23, Switzerland
}

November 12, 1992

In this talk I would like to report on joint work done in collaboration with J.L.F. Barbón and $\check{C}$. Crnković ${ }^{1}$, aiming at finding an exact and tractable formulation of non-critical string theory in space-time dimensions $D>1$. The coupling of $D \leq 1$ conformal field theories to two-dimensional gravity has been successfully studied using continuum methods ${ }^{2,3,4,5}$ and matrix models $s^{6,7,8,9}$. For $D>1$, apart from the original papers ${ }^{6,7}$ and the work of Boulatov et al. ${ }^{10}$, little work has been done analytically to understand these systems. An interesting exception is Ref. 11. In our own work, we use techniques of large- $N$ Quantum Field Theory to reduce the exact partition function for strings in $D>1$ (but $D<6$ ) to a single Hermitian one-matrix problem. The reduced model techniques of Eguchi-Kawai ${ }^{12}$ (EK) and the Twisted Eguchi-Kawai construction of Gonzalez-Arroyo and Okawa ${ }^{13,14}$ allow us to obtain an exact correspondence with certain types of one-matrix models. By analyzing the effective actions derived from these matrix models, we identify a family of critical points whose string susceptibilities are characterized by two integers $(n, m)$ and such that

$$
\gamma_{s t}=\frac{p}{n+m+1} 0 \leq p \leq n
$$

We find a second-order phase transition where both gravity and some polymer-like matter become critical simultaneously. It is at this multicritical point that we take the continuum limit yielding the exponents (1). We have also computed the planar correlation functions of a collection of macroscopic loop operators. When $n=1, m \geq 2$, the general structure of these correlators seems to indicate that our approximations to the exact model do capture some of the basic features of tachyonic states.

The basic problem in describing the motion of strings in D-dimensional space-time is the evaluation of the functional integral:

$$
\begin{aligned}
\mathcal{Z} & =\int \mathcal{D} g_{a b} \mathcal{D} X_{\mu} \\
\exp & \left(-\frac{1}{2} \int_{\Sigma} d^{2} \sigma \sqrt{g} g^{a b} \partial_{a} X^{\mu} d b X_{\mu}-\right. \\
& \left.-\mu_{0} \int_{\Sigma} \sqrt{g}+\ldots\right)
\end{aligned}
$$

where $X^{\mu}$ is the field representing the embedding of the string world-sheet into space-time, and $g_{a b}$ is the two-dimensional metric. The partition function (2) is quite formal, and one can regularize $i t^{6,7,8,9}$ by replacing the sum over two-dimensional geometries by a sum over triangulations. The result is:

$$
\begin{aligned}
\mathcal{Z} & =\sum_{T} \frac{e^{-\mu|T|}}{n(T)} \int \prod_{i \in T_{0}} \sigma_{i}^{\alpha} d^{D} X_{i} \\
\cdot & \prod_{\langle i j\rangle \in T_{1}} G\left(X_{i}-X_{j}\right)
\end{aligned}
$$

In (3) $\mu$ is the bare two-dimensional cosmological constant, $T$ is a generic triangulation, $T_{0}$ (resp. $T_{1}$ ) represents the set of vertices (resp. edges) of $T, \sigma_{i}=q_{i} / 3$, where $q_{i}$ is the coordination number of site $i$, and $G\left(X_{i}-X_{j}\right)$ is a propagator factor. To reproduce (2), we take $G(X)$ to be a Gaussian. Using large- $N$ 
techniques, it is shown in Refs. 8 and 9 that $\mathcal{Z}$ is equivalent to the large- $N$ limit of a $N \times N$ $\Phi^{3}$ matrix field theory:

$$
\begin{aligned}
\mathcal{Z} & =\lim _{N \rightarrow \infty} \log \int \mathcal{D} \Phi \\
\exp & \left(-N \int d^{D} X \operatorname{Tr}\left(\frac{1}{2} \phi G^{-1} \phi+\right.\right. \\
+ & \left.\left.\frac{1}{3} g \Phi^{3}\right)\right) ; g=e^{-\mu}
\end{aligned}
$$

Since for $D<6$ the $\Phi^{3}$ field theory is superrenormalizable, the critical properties of (4) should not depend on whether we replace the Gaussian by the Feynman propagator in (4). We will assume this replacement from now on. Writing $\mathcal{Z}$ in terms of the free energy $F, \mathcal{Z}=\exp N^{2} F$, we are interested in finding the critical values $g_{c}$ for the cosmological constant. Near these values we find the scaling behaviour:

$$
\chi \sim \frac{\partial^{2} F}{\partial g^{2}} \sim\left(g-g_{c}\right)^{-\gamma_{s t}}
$$

To prove the equivalence of (4) to a onematrix model, we first take a lattice approximation for the D-dimensional target spacetime with periodic boundary conditions. This lattice will have $L^{D}$ sites. The next step is to note that for $S U(N)$ we can construct a collection of $D$ twist matrices $\Gamma_{\mu}, \mu=1,2, \ldots, D$ commuting up to an element of the centre of $S U(N), Z_{N}$ :

$$
\begin{gathered}
\Gamma_{\mu} \Gamma_{\nu}=\mathcal{Z}_{\nu \mu} \Gamma_{\mu} \Gamma_{\nu} \\
\mathcal{Z}_{\mu \nu}=e^{Z_{M 2} n_{\mu \nu} / N}
\end{gathered}
$$

$n_{\mu \nu}=-n_{\nu \mu}$ is a matrix of integers. One of the basic ideas of the TEK reduction ${ }^{13}$ is to require the $\Gamma_{\mu}$ 's to provide a faithful projective representation of the $L^{D}$-lattice translation group. This implies some constraints on $n_{\mu \nu}$, among them $N=L^{D / 2}$. Then in the large- $N$ limit, following Refs. 12 and 13, we can show that the planar Schwinger-Dyson equation for (4) coincides with those of the reduced theory obtained from (4) by replacing:

$$
\Phi(x) \rightarrow D(x) \phi D(x)^{-1}
$$

$$
D(x)=\prod_{\mu=1}^{D} \Gamma_{\mu}^{x_{\mu}}
$$

The $x_{\mu}$ 's are measured in integer units of the lattice spacing. The reduced action is therefore:

$$
S=\frac{1}{2} \sum_{\mu} \operatorname{Tr}\left(\Gamma_{\mu} \Phi \Gamma_{\mu}^{-1}-\Phi\right)^{2}+\frac{1}{3} g \operatorname{tr} \Phi^{3}
$$

where now $\Phi$ is a constant matrix. One important property of $(10)$ is that all its planar graphs coincide graph by graph with those of the original theory. We can go further and simply take (10) as our definition of the Ddimensional non-critical bosonic string. As constructed, (10) only works in $D=2,4$. However, some simple modifications allow us to construct theories in $D=3,5$ (see Ref. 1 for details).

The obvious thing one would like to do with (10) is to write $\Phi$ in terms of a unitary matrix $U$ and a real diagonal matrix $\gamma$ :

$$
\Phi=U\left(\begin{array}{cc}
\gamma_{1} & \\
\ddots & \\
& \gamma_{N}
\end{array}\right) U^{+}
$$

and to perform subsequently the angular integral (integration over U). The result should be an effective one-matrix model of the form:

$$
\begin{gathered}
\mathcal{Z}_{e f f}=\int D \Phi e^{-N^{2} \Gamma_{e f f}[\Phi]} \\
\Gamma_{e f f}[\Phi]=V\left(x_{1}, x_{2}, \ldots, x_{n}, \ldots\right)+\sum_{i \geq 1} g_{i} x_{i} \\
x_{n} \equiv \frac{1}{N} \operatorname{Tr} \phi^{n}
\end{gathered}
$$

where $V$ is a function at least quadratic in the trace variables $x_{n}$. Note further that the vertices $g_{n} x_{n}$ with standard large- $N$ techniques generate simplicial approximations to smooth surfaces. On the other hand, couplings of the form $x_{n_{1}} x_{n_{2}}, \ldots, x_{n_{p}}$ represent $p$ triangulated surfaces touching at one point, where the number of double lines contributed to the diagram by $x_{n_{i}}$ equals $n_{i}$. Couplings of the form $x_{2}^{2}$ were first studied in Ref. 15. These couplings, if 
they dominate, tend to make the surface look like a collection of branched polymers. From the point of view of a continuum formulation, these couplings can be interpreted in terms of singular configurations of the Liouville field. Inclusion of these singularities in the continuum quantization of Liouville theory is yet to be done. The singularities would correspond to making the metric vanish along some random gas of closed curves. In the simplest case of $x_{2}^{2}$ square interactions, these curves of vanishing metric should be self-avoiding. In Ref. 1 we have been able to solve exactly (13) in the planar limit by assuming that $V$ is an analytic function of the $x_{i}$ 's and with the added simplification that $V$ depends on an arbitrary, although finite, number of $x_{i}$ 's. Furthermore, and always under these assumptions, we have determined under what conditions there is "enough room" to open macroscopic loops on the surface. In those cases where this is possible, we have computed all planar multiloop correlation functions. We now briefly outline the salient features of our analysis. We find three types of phases:

1. $\gamma_{s t}<0$. The gravity couplings become critical, and the polymer couplings are non-critical. This is essentially the same as the pure gravity case.

2. $\gamma_{s t}>0$. Gravity is not critical, but matter becomes critical. This case describes a phase akin to branched polymers.

3. $\gamma_{s t}>0$. An interesting subset of item two, both gravity and matter become critical simultaneously. This is the multicritical point in which we are interested. In this case $\gamma_{s t}$ takes the values given in (1) depending on details. The phase transition is second order, and it makes sense to go to the continuum limit. In the pair of integers $(n, m)$ determining the string susceptibility in (1), $n$ gives the order of criticality in the polymer couplings and $m$ gives it in the pure gravity couplings. $n=1$ corresponds to tuning only the quadratic part of $V$ in (13).
4. We have computed the correlation functions of macroscopic loops for the $(n, m)$ critical points. We find that only for $n=1$ can we open them. For other cases $(n>1)$, we could say naively that the polymerization is so complete that there is hardly any room to open up those loops on the surface. For $(1, m)$ theories, the two-loop correlator is given by:

$$
\begin{array}{r}
\left\langle w\left(\ell_{1}\right) w\left(\ell_{2}\right)\right\rangle=\frac{e^{-\left(\ell_{1}+\ell_{2}\right) / u}}{\ell_{1}+\ell_{2}}+ \\
+\frac{u}{\ell_{1} \ell_{2}} e^{-\left(\ell_{1}+\ell_{2}\right) / n},
\end{array}
$$

and $u \propto \mu^{-\frac{1}{2}}$, where $\mu$ is the renormalized cosmological constant in the simple case. Note that the second term on the righthand side can also be written as:

$$
\left\langle w\left(\ell_{1}\right) P\right\rangle \frac{1}{\langle P P\rangle}\left\langle P w\left(\ell_{2}\right)\right\rangle,
$$

where $P$ is the puncture operator. This contribution is reminiscent of the tachyon contribution in critical string theories. Similar results are obtained for multiloop correlators. The fact that the puncture operator $P$ behaves in some sense as a tachyon state gives some credence to the approximation of $V$ we have taken. Obviously more work is required before we can definitely conclude that our truncated effective action captures the universal features of the original model (10).

5. We have computed the $1 / N^{2}$ corrections to our model and they are in agreement with the genus dependence found by David $^{4}$ and Distler and Kawai ${ }^{5}$.

We feel that features 1 - 5 provide encouraging evidence that our approximate study of the original model (10) captures some of its universal features. More work is certainly necessary to determine the critical properties of (10). Further details can be found in Ref. 1.

\section{ACKNOWLEDGEMENTS}


I would like to thank Professors E. Rabinovici and A. Schellekens for the opportunity to present this work at this conference.

\section{REFERENCES}

1. L. Alvarez-Gaumé, J.L.F.Barbón and C. Crnković, "A Proposal for $D>$ 1 Strings", CERN preprint CERNTH.6600/92.

2. A.M. Polyakov, "Quantum Gravity in Two Dimensions", Mod. Phys. Lett. A2, p. 893 (1987).

3. V.G. Knizhnik, A.M. Polyakov and A.B. Zamolodchikov, "Fractal Structure of 2D Quantum Gravity", Mod. Phys.Lett. A3, p. 819 (1988).

4. F. David, "Conformal Field Theories Coupled to 2D Gravity in the Conformal Gauge", Mod. Phys. Lett. A3, p. 1651 (1988).

5. J. Distler and H. Kawai, "Conformal Field Theory and 2D Gravity", Nucl. Phys. B321, p. 509 (1988).

6. J. Ambjørn, B. Durhuus and J. Fröhlich, "Diseases of Random Triangulated Surface Models and Possible Cures", Nucl. Phys. B257, p. 433 (1985).

7. J. Ambjørn, B. Durhuus, J. Fröhlich and P. Orland, "The Appearance of Critical Dimensions in Regulated String Theories", Nucl. Phys. B270, p. 457 (1986).

8. F. David, "Planar Graphs, 2D Gravity and Surface Models", Nucl. Phys. B257, p. 53 (1985);

"A Model of Random Surfaces with NonTrivial Critical Behaviour", Nucl. Phys. B257, p. 543 (1985).

9. V. Kazakov, "Bilocal Regularization of Models of Random Surfaces", Phys. Lett. 150B, p. 282 (1985).
10. D. Boulatov, V.A. Kazakov, I. Kostov and A.A. Migdal, "Analytic and Numerical Study of Dynamically Triangulated Random Surfaces", Nucl. Phys. B275, p. 641 (1986).

11. E. Brézin and S. Hikami, "A Naive Matrix Model Approach to Two-Dimensional Quantum Gravity Coupled to Matter of Arbitrary Central Charge", preprint LPTENS 92/10.

12. T. Eguchi and H. Kawai, "Reduction of Degrees of Freedom in the Large N Gauge Theory", Phys. Rev. Lett. 48, p. 1063 (1982).

13. A. Gonzalez-Arroyo and M. Okawa, "The Twisted Eguchi-Kawai Model, a Reduced Model for Lattice Gauge Theory", Phys. Rev. D27, p. 2397 (1983).

14. For a review of EK and TEK models, see: S.R. Das, "Some Aspects of Large N Theories", Rev. Mod. Phys. 59, p. 235 (1987).

15. S.R. Das, A. Dhar, A.M. Sengupta and S.R. Wadia, "New Critical Behaviour in $\mathrm{d}=0$, Large N Matrix Models", Mod. Phys. Lett. A5, p.1041 (1990). 